\title{
A case report of pyomyoma: radiological diagnosis of a potentially fatal complication of uterine leiomyoma.
}

\author{
Mubarak MY, MMED (Radiology) ${ }^{\mathrm{a}}$, Noordini MD, MMED (Anaesthesiology) ${ }^{\mathrm{b}}$ \\ a Department of Diagnostic Imaging, b Department of Anaesthesiology and Intensive Care, \\ Hospital Tengku Ampuan Afzan, Malaysia.
}

\begin{abstract}
Pyomyoma (suppurative leiomyoma) is a rare complication of uterine leiomyoma. It results from infarction and infection of a leiomyoma. It may lead to diagnostic and therapeutic difficulties with potential complications such as bacteraemia, uterine rupture, compressive effect to adjacent structures and even death. We report a case of pyomyoma based on the Ultrasound (US) and Computed Tomography (CT) findings and literature review on the subject.
\end{abstract}

KEYWORDS: Pyomyoma, Leiomyoma, Ultrasound, Computed Tomography.

\section{INTRODUCTION}

Pyomyoma is a rare but serious infectious complication of uterine leiomyoma. The diagnosis of pyomyo$\mathrm{ma}$ is difficult because of inadequate specific clinical and reported radiological findings. The presence of fluid and gas in pre-existing uterine leiomyoma on abdominal ultrasound (US) and computed tomography (CT) may lead to the diagnosis.

\section{CASE REPORT}

A fifty-two year old woman was diagnosed to have uterine leiomyoma for three years. The leiomyoma was gradually increasing in size. She presented with abdominal pain and bilateral lower limb swelling for one month and fever for two weeks. She had loss of weight and appetite for the past few months. Abdominal mass was palpable on clinical examination and she was subjected to an US Scan of the abdomen. The scan revealed a huge pelvic mass measuring $13.5 \mathrm{~cm} \mathrm{x}$ $15.0 \mathrm{~cm}$. The mass was hypoechoic with internal echogenic debris and reverberation artefacts produced by gas centrally [Figure 1]. The urinary bladder was inferior and anterior to the mass. The normal uterus was not visualized. Both kidneys were hydronephrotic with bilateral proximal hydroureters. Based on the US

\section{Correspondence Author:}

Mubarak Mohd Yusof, Department of Diagnostic Imaging

Hospital Tengku Ampuan Afzan,

Jalan Tanah Putih,

25100 Kuantan,

Pahang Darul Makmur,

Malaysia.

Tel: +6095572056 ,

Fax: +6095149396 ,

email: adibawazif@yahoo.com and clinical presentation, a diagnosis of an infected mass arising from the uterus with bilateral obstructive uropathy was made. A differential diagnosis of uterine sarcoma was also given.

An enhanced helical CT scan of the abdomen and thorax were performed to evaluate the abdominal mass and to look for possible metastasis. Ring enhancing lesions due to abscesses were visualized in the spleen and right psoas muscle. The pelvic mass measured $13 \mathrm{~cm} \times 16.5 \mathrm{~cm} \times 19.2 \mathrm{~cm}$ and originated from the uterus. It has thick wall, cystic component and gas [Figure 2]. There was a cystic collection on the left of the urinary bladder [Figure 3]. Thromboses were noted in the right and left femoral veins. The mass was diagnosed to be a pyomyoma. CT scan of the thorax showed few thromboses in the right and left pulmonary arteries consistent with pulmonary embolism. There was no mass in the mediastinum and lung parenchyma.

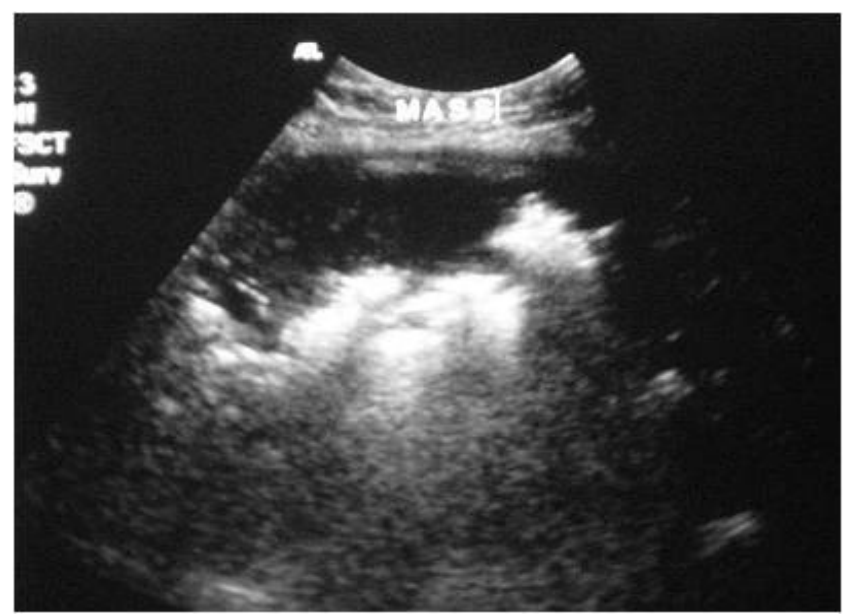

Figure 1. Transverse sonogram of the uterine mass showing internal echoes and reverberation artefact from gas suggestive of pyomyoma. 


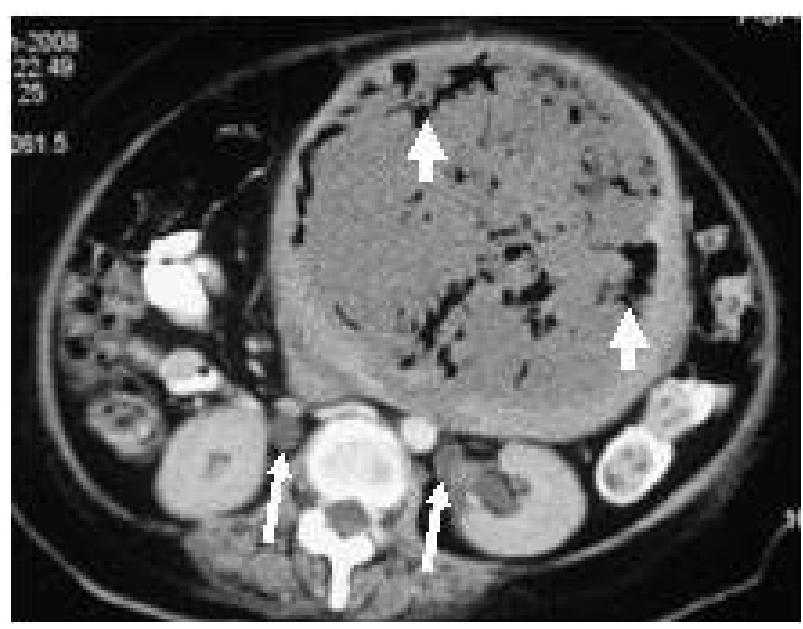

Figure 2. CT of pyomyoma showing the fluid filled content with collection of air within it. Dilated ureters are shown (long arrows).
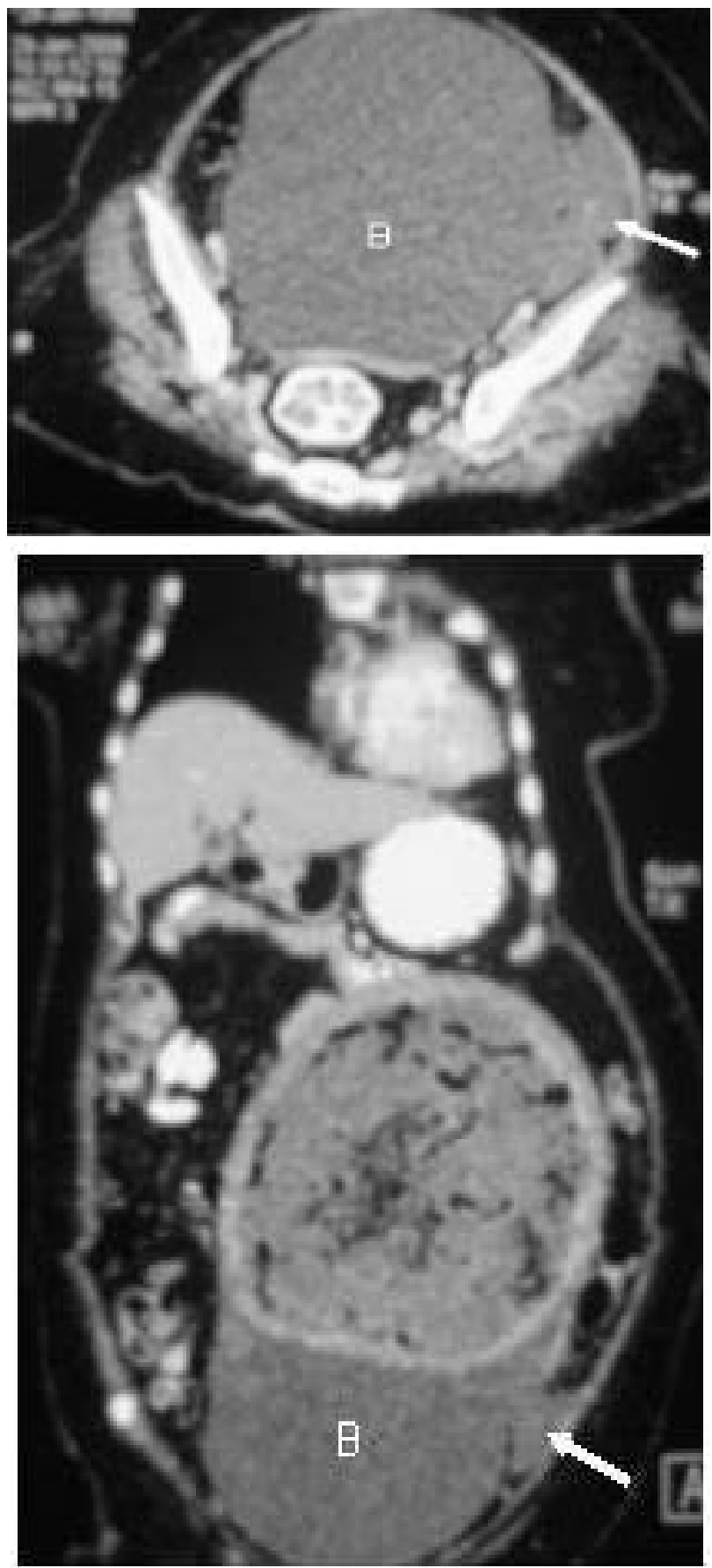

Figure 3. (a) Axial CT image revealed distended bladder, B, and collection lateral to the bladder (arrow) which suggest the pyomyoma may have ruptured. (b) Coronal reconstruction image shows the mass, bladder, B, and the collection (arrow).

The urine and blood cultures and tumour markers were unremarkable. The patient was diagnosed to have diabetes mellitus and acute renal failure with serum creatinine ranging from $378 \mu \mathrm{mol} / \mathrm{L}$ to $535 \mu \mathrm{mol} / \mathrm{L}$ during this admission. She was put on haemodialysis and was treated with insulin, intravenous anti-coagulant and antibiotics. An attempt was made to percutaneously aspirate and drain the abscess in the leiomyoma under US guidance but it failed due to the thickened wall of abscess. The patient's clinical condition deteriorated and she succumbed after about one month in the high dependency unit due to sepsis and renal failure.

\section{DISCUSSION}

Uterine leiomyomas are the most common neoplasm of the uterus. They are derived from the smooth muscle cells and most patients are asymptomatic. They can present with a palpable mass, per vaginal bleeding, pain and symptoms due to compression of the mass on the bladder and rectum. The secondary changes of leiomyoma are hyaline degeneration, cystic degeneration, calcification, fatty degeneration, necrosis and sarcomatous transformation. 1

There are many complications associated with leiomyoma. They include nephrotic syndrome, hydronephrosis, deep venous thrombosis and suppuration.2 Pyomyoma is a rare but potentially fatal complication. It is thought to occur from infarction and infection of a leiomyoma. It is more common in submucosal leiomyoma because the blood supply is inadequate for the tumour mass. 1 The central portion of the mass might have purulent material or gas.1, 3 Pyomyoma can also occur after uterine artery embolization of leiomyoma. 4 The common symptoms of a pyomyoma are pelvic pain, fever and weight loss. The time of diagnosis can be delayed up to one year from the onset of the symptoms. 2

There are less than one hundred cases of pyomyoma reported in the literature and most cases occurred prior to 1945 , before the era of antibiotics.2, 3 Sonographic findings of pyomyoma include an enlarging pelvic mass with solid or cystic component. The presence of gas in leiomyoma is diagnostic of pyomyoma. The gas as in our case produce reverberation artefact which was also reported in the literature.3 CT scan findings of pyomyoma would include heterogeneous density mass with the presence of gas and debris. In our case, the "black" areas within the mass have density of gas (Hounsfield Unit -457 to -535 ). The fluid has density of purulent material (Hounsfield Unit +23 to +35.8$)$. The cystic collection immediately to the 
left of the bladder has density of purulent material and could be an evidence of rupture from the pyomyoma. 3

The treatment of pyomyoma will include intravenous antibiotics, myomectomy or hysterectomy in addition to the anti-coagulant therapy for deep vein thrombosis in our patient. There was no report regarding percutaneous aspiration in pyomyoma. The causative organisms could be isolated from the blood or the mass. Unfortunately there was no organism cultured from the urine and blood of our patient. She was not subjected for surgery in view of her poor clinical conditions. In conclusion, because of its rarity, pyomyoma can be difficult to diagnose especially if one is not aware of this condition and of its appearances on the US and CT scan.

\section{REFERENCES}

1. Casillas J, Joseph RC, Guerra JJ Jr. CT Appearance of Uterine Leiomyomas. Radiographics 1990; 10:999-1007.

2. Genta PR, Dias ML, Janiszewski TA, et al. Streptococcus agalacticae endocarditis and giant pyomyoma simulating ovarian cancer. South Med J 2001; 94:508-11.

3. Karcaaltincaba M, Sudakoff GS. Case Report: CT of a Ruptured Pyomyoma. Am J Roentgenol 2003; 181:1375-77.

4. Kitamura Y, Ascher SM, Cooper C, et al. Imaging Manifestations of Complications Associated with Uterine Artery Embolization. Radiographics 2005; 25:S119-32. 